\title{
Cavete Collegae: Erosion des Strahlenschutzes!
}

\begin{abstract}
Claudio Knüslia, Martin Walter ${ }^{b}$, Andreas Nidecker $^{c}$, Jacques Schiltknecht ${ }^{d}$, Jacques Moser ${ }^{\text {, }}$ Jean-Jacques Fasnacht ${ }^{\mathrm{e}}$, Bettina Wölnerhanssen ${ }^{f}$, für den Vorstand PSR/IPPNW Schweiz

${ }^{a}$ Dr. med. Innere Medizin / Onkologie FMH; ${ }^{b}$ Dr. med. Innere Medizin FMH; ${ }^{c}$ Prof. (em.) Dr. med. Radiologe FMH; ${ }^{d}$ Dr. med. Innere Medizin FMH;

${ }^{e}$ Dr. med. Allgemeine Medizin FMH; ${ }^{f}$ PD Dr. med. klinische Forschung/Chirurgie FMH
\end{abstract}

Die massive Erhöhung des Dosisgrenzwertes bei der durchgeführten Revision von Verordnungen zum Kernenergiebereich [1] heizt die Diskussion um die Sicherheit der Bevölkerung im Einzugsbereich des weltweit dienstältesten Kernkraftwerkes in Beznau/AG an. Im Zentrum steht das Gesundheitsrisiko durch störfallbedingte Exposition der Menschen gegenüber ionisierender Strahlung im niedrigen Dosisbereich.

\section{Wissenschaftliche Fakten zu strahlen- induzierten Gesundheitsfolgen}

Die Erkenntnisse über strahleninduzierte Krankheiten aus den systematischen Untersuchungen an japanischen Atombombenüberlebenden bilden die Basis der Risikoeinschätzung im heutigen medizinischen Strahlenschutz [2]. Ergänzend sind in den letzten Jahren mindestens acht umfassende epidemiologische Stu-

\section{Zusammenfassung}

Studien zu japanischen Atombombenüberlebenden, aus Arbeitsmedizin und Medizindiagnostik sowie zur natürlichen Strahlung bilden die wissenschaftliche Grundlage für die geltenden Grenzwerte für ionisierende Strahlen. Doch obschon der Strahlenschutz auf der Epidemiologie und der Strahlenbiologie basiert, steht er mehr denn je im Spannungsfeld von Medizin, Wirtschaft und Politik. Zur Risikoabschätzung von strahleninduzierten Krebserkrankungen dient das Linear-no-Threshold-(LNT-)Modell. Es besagt, dass hinsichtlich Krebsrisiko eine Dosis-Wirkungs-Beziehung ohne Schwellenwert besteht. Moderne Untersuchungen bestätigen die Gültigkeit des LNT-Modells auch für Strahlendosen unter 100 Millisievert (mSv). Der Strahlenschutz wird aktuell - selbst in medizinischen Fachzeitschriften durch Autoren diskreditiert, die der Nuklearindustrie nahestehen. Ärztinnen und Ärzte seien darauf aufmerksam gemacht. Ebenso sind das Eidgenössische Nuklearsicherheitsinspektorat ENSI sowie die Entscheidungsträger dringend aufgerufen, ärztliche Bedenken ernst zu nehmen. Eine Erhöhung des Dosisgrenzwertes für geplante Expositionssituationen um einen Faktor 100, wie sie die Revision der Verordnungen zum Kernenergiebereich vorsieht, ist unverantwortlich und muss aus präventivmedizinischer Sicht klar abgelehnt werden. dien publiziert worden, die zeigen, dass diese Risikoeinschätzung auch für Strahlenexpositionen im Dosisbereich von 1-100 mSv Gültigkeit hat (Tab. 1). Die mehrfach reproduzierte Beobachtung einer Dosis-Wirkungs-Beziehung durch verschiedenste, voneinander unabhängig arbeitende Forschungsgruppen ist eines der härtesten Kriterien für Kausalität: Das Krebserkrankungsrisiko steigt linear mit der Expositionsdosis. Diese Studien bestätigen somit das Konzept des Linear-no-Threshold-(LNT-)Modells [3]. Der schweizerische Bundesrat teilt diese Einschätzung der Bedeutung niedriger ionisierender Strahlendosen in seiner Medienmitteilung vom 2. März 2018 [4a, b].

\section{Übersicht: Studien zu Gesundheitseffek- ten bei niedrigen Strahlendosen (Tab. 1)}

- In der INWORKS-Studie [5-7] wurden über 300000 Nukleararbeiter aus Frankreich, Grossbritannien und den USA über die Dauer von mehr als 20 Jahren untersucht. Die Strahlenexposition wurde dosimetrisch erfasst und betrug durchschnittlich $20 \mathrm{mSv}$. Es fand sich ein signifikant erhöhtes Mortalitätsrisiko für solide Neoplasien, für Leukämien wie auch für nicht maligne Erkrankungen (kardiovaskuläre Todesfälle).

- Die Studie von Darby [8] aus 9 europäischen Ländern zeigte, dass die Lungenkarzinommortalität mit zunehmender Radonexposition in Häusern ab dem Dosisbereich von 3-4 mSv/Jahr ansteigt.

- Studien bei Kindern und Jugendlichen aus Grossbritannien [9] und Australien [10] ergaben über- 
Tabelle 1.

Epidemiologische Studien mit statistisch signifikanter, dosisabhängiger Häufung von Gesundheitsschäden (Inzidenz u./o. Mortalität) bei niedrigen Dosen ionisierender Strahlung

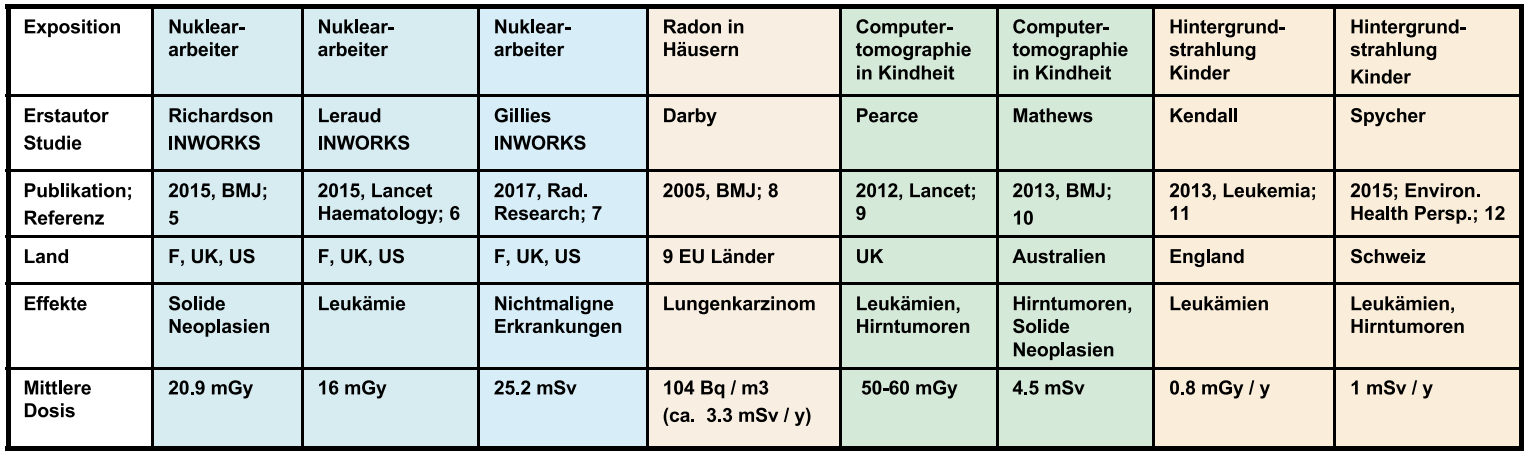

IPPNW.ch 2018

Tabelle 1: Übersicht Studien zu Gesundheitseffekten bei niedrigen Strahlendosen.

einstimmend eine Zunahme der Leukämie- und Hirntumorinzidenz nach Computertomographien (Strahlendosen um durchschnittlich 5-60 mSv) in der Kindheit und Jugend.

- Zwei Studien - aus Grossbritannien [11] sowie der Schweiz [12] - untersuchten die Krebshäufigkeit bei Kindern in Abhängigkeit von der natürlichen Hintergrundstrahlung im Bereich von $1 \mathrm{mSv}$ pro Jahr. Kendall fand eine signifikante, dosisabhängige $\mathrm{Zu}$ nahme kindlicher Leukämien, und auch die Arbeitsgruppe von Spycher am Institut für Sozial- und Präventivmedizin der Universität Bern beobachtete eine Zunahme der Inzidenz von Leukämien und Hirntumoren bei Kindern selbst bei diesen sehr niedrigen Strahlendosen [12].

\section{Unqualifizierte Angriffe auf den Strahlen- schutz nehmen zu}

In den vergangenen Monaten ist eine zunehmende publizistische Tendenz zu beobachten, den Strahlenschutz in seinen Grundsätzen in Frage zu stellen und

\section{Bei Gesundheitsrisiken ionisierender Strahlen von einer Schwelle zu sprechen ist abwegig.}

die Risiken ionisierender Strahlung zu verharmlosen. Provoziert wird dies unter anderem durch einen Erlass der US-Regierung von Anfang 2017 [13], die eine Aufweichung der Strahlenschutzgesetzgebung aus wirtschaftlichen Gründen im Interesse der Nuklearindustrie ins Auge fasst [14]. Die Autoren sind oft Physiker, die Letzterer nahestehen und die sich an den geltenden Strahlenschutzbestimmungen stossen [14-18]. Diese stellen die Nuklearindustrie bei der Entsorgung von radio- aktiven Materialien aus Kernkraftwerken vor kostenintensive Aufgaben. Einen Ausweg sehen die Autoren in der Lockerung der Strahlenschutz-Grenzwerte. Dabei wird die heute weltweit von den massgebenden Strahlenschutzgesellschaften akzeptierte Risikobeurteilung für Gesundheitsschäden durch ionisierende Strahlung willkürlich für ungültig erklärt. Teilweise finden sich - selbst in medizinischen Fachzeitschriften - bizarre Vorstellungen, so zum Beispiel, dass radiodiagnostische Strahlenexposition die Prognose verbessere [15] oder dass eine «Radiophobie» der Ärzteschaft zu insuffizienter Radiodiagnostik führe, was letztlich bei Patienten eine schlechtere Prognose zur Folge habe [16]. Ferner wird für eine Renaissance der längst verlassenen Hormesishypothese («Lebensverlängerung durch Radioaktivität») auf der Basis frei erfundener Untersuchungsresultate geworben [17, 18], was sich auch unreflektiert in der Tagespresse niederschlägt [19].

\section{Eidgenössisches Nuklearsicherheits- inspektorat (ENSI) auf Abwegen}

Auch die nachfolgend zitierte Stellungnahme der Leiterin des Fachbereiches Strahlenschutz des ENSI, der Nuklearingenieurin Frau Rosa Sardella, von Anfang Februar 2018 muss abgelehnt werden: «Die Strahlendosis, die bei einem 1000-jährlichen Erdbebenereignis maximal erlaubt ist [ $1 \mathrm{mSv} / \mathrm{Jahr}$; Autor], liegt weit unterhalb der Schwelle, die für Mensch und Umwelt schädlich sein könnte» [20a]. Bei Gesundheitsrisiken ionisierender Strahlen von einer Schwelle zu sprechen ist abwegig, denn dies widerspricht den heute geltenden, wissenschaftlichen Grundlagen des Strahlenschutzes. Fragwürdig ist ebenfalls die weiter angehängte Formu- 
lierung «Auch bei einer solchen Dosis [von 100 Millisievert; Autor] können keine deterministischen, beziehungsweise unmittelbar feststellbaren gesundheitlichen Effekte festgestellt werden; das Krebsrisiko, das heisst die Wahrscheinlichkeit für einen Schaden, steigt minim an» [20a]. Diese Erwähnung des Fehlens deterministischer Effekte (wie beispielsweise Strahlenerythem) bei einer Dosis von $100 \mathrm{mSv}$ lenkt vom Thema ab, geht es doch zum Vornherein um die stochastischen (zufallsbedingten, jedoch dosisabhängigen), häufig letalen Strahlenfolgen. Deren quantitative Wertung durch das ENSI («minimer Anstieg des Krebsrisikos») bedarf im Folgenden einer eingehenderen Betrachtung.

\section{Strahlungsbedingter «minimer Anstieg des Krebsrisikos»?}

Das absolute Risiko (EAR; excess absolute risk), an einem durch ionisierende Strahlung verursachten Krebs $\mathrm{zu}$ versterben, wird in den aktuell geltenden Richtlinien der Internationalen Strahlenschutzkommission (ICRP) von 2007 mit 5\%/Sievert innerhalb von 50 Jahren angegeben [21]. Da die Krebsinzidenz etwa doppelt so hoch wie die Krebsmortalität zu veranschlagen ist, entspricht dies bei einer Dosis von 100 Millisievert einem strahlungsbedingten Krebserkrankungsrisiko von $1 \%$. Es ist augenfällig, dass die Bedeutung dieses Risikofaktors von der Expositionssituation abhängt: Handelt es sich um eine individualmedizinische, klinisch-diagnostische Patientensituation oder um eine präventivmedizinische Fragestellung bei der Abschätzung der Strahlenrisiken bei grossen Kollektiven gesunder Menschen?

- Als Ärztinnen und Ärzte stützen wir uns im Alltag bei der Betreuung des einzelnen Patienten auf Richtlinien der Fachgesellschaften, die dem medizinischen Strahlenschutz Rechnung tragen [22]. Das genannte Zusatzrisiko durch die Radiodiagnostik wird dabei gegenüber dem diagnostischen Erkenntnisgewinn abgewogen. Die Verantwortung liegt bei den verordnenden Ärztinnen und Ärzten.

- Im Kontext der Kernenergiegesetzgebung bezieht sich der Strahlenschutz jedoch auf die Prävention bei einer Bevölkerung von Zehntausenden bis vielen Hunderttausenden gesunden Individuen, die im Falle eines grösseren Kernkraftwerkunfalls strahlenexponiert sind. So ist bei einer störfallbedingten Verstrahlung von 100000 gesunden Personen mit einer Dosis von je 100 mSv mit 1000 strahleninduzierten Krebserkrankungen innerhalb 50 Jahren zu rechnen. Zusätzlich muss (in einer Häufigkeit von mindestens derselben Grössenordnung) von strahlungsinduzierten lebensbedrohlichen kardiovasku- lären und anderen nichtonkologischen Erkrankungen, ferner auch von genetischen Schädigungen ausgegangen werden [2, 7, 23]. Mit anderen Worten: Bei einer Verstrahlung der Bevölkerung im Dosisbereich um $100 \mathrm{mSv}$ in den Zonen $1+2$ der KKW Beznau/Leibstadt (20-km-Radius, überlappende Zonen [24], Gebiete in Deutschland nicht eingerechnet) mit aktuell 286824 Einwohnern müsste strahlungsbedingt mit über 2800 Todesopfern (vorzeitige Todesfälle infolge von Krebs- und nichtmalignen Erkrankungen) und zusätzlich Tausenden ernsthaft Erkrankten gerechnet werden. Hier kann mit Sachkenntnis und gesundem Menschenverstand nicht mehr von «minimen Risiken" gesprochen werden.

\section{Weshalb eine Revision der Verordnungen im Kernenergiebereich?}

Nach der nuklearen Katastrophe von Fukushima infolge des Erdbebens und Tsunamis vom 11.3.2011 stellte das ENSI bei einer Risikoanalyse fest, dass die Bevölkerung bei einem Erdbeben, wie es hinsichtlich Stärke alle 10000 Jahre auch in der Schweiz vorkommen kann, durch Schäden im KKW Beznau mit einer Dosis von 28.9 bis $78 \mathrm{mSv}$ verstrahlt würde [25]. Diese hohen

\section{Diese Gesetzwidrigkeit muss eine Ausser- betriebnahme des KKW Beznau zur Folge haben.}

Werte verstossen gegen die geltende Strahlenschutzverordnung, die bei einem Erdbeben dieser Grössenordnung $\left(10^{-4}\right)$ eine zusätzliche Strahlenbelastung von nur $1 \mathrm{mSv}$ zulässt. Diese Gesetzwidrigkeit muss eine Ausserbetriebnahme des KKW Beznau zur Folge haben. Einen Ausweg suchen die Behörden mit einer Revision, sprich 100-fachen Erhöhung des zulässigen Dosisgrenzwerts (von $1 \mathrm{mSv}$ auf $100 \mathrm{mSv}$ ) für die «Erdbebenkategorie $10^{-4}$ ", sowie der Schaffung einer neuen Erdbebenkategorie mit Wahrscheinlichkeit des Eintretens von $10^{-3}$ (1 Mal pro 1000 Jahre). Die Legitimation zu diesem Schritt, der einer massiven Schwächung des Strahlenschutzes entspricht, sieht der Bundesrat in unklaren und missverständlichen Formulierungen mehrerer Verordnungen [26].

Es ist jedoch in keiner Weise nachvollziehbar, weshalb diese offenbar revisionsbedürftigen Formulierungen $\mathrm{zu}$ einer neuen Kategorisierung $\left(10^{-3}\right)$ führen und insbesondere der interessierende Wert $\left(10^{-4}\right)$ aus der «Störfallkategorie zwischen $10^{-2}$ und $10^{-4}$ » entfernt werden soll, welche der Bevölkerung bisher den gesetzlichen Schutz der maximal zulässigen Dosis von $1 \mathrm{mSv}$ zugesichert hat. Besonders stossend ist daran, dass das 
ENSI sich in dieser Situation nicht an die von ihm selber wörtlich bereits im Jahre 2009 festgehaltenen Regeln hält: «Falls die Zuordnung zu einer Störfallkategorie nicht eindeutig ist, ist der Störfall im Sinne der Vorsicht der tieferen Kategorie (mit den strengeren Anforderungen) zuzuweisen» [27].

\section{Alternde Kernreaktoren und Lockerung des Strahlenschutzes - wie passt das zusammen?}

Es liegt auf der Hand, dass die vorgesehene Grenzwerterhöhung im Rahmen der Revision der Verordnungen im Kernenergiebereich nicht im Interesse der Bevölkerung sein kann. Gerade bei uns Ärztinnen und Ärzten mit präventivmedizinischer Verpflichtung müssen die Alarmglocken läuten. Wir kennen die Herausforderung steigender gesundheitlicher Umweltrisiken im Verlaufe der Zeit. Wir sind es gewohnt, in solchen Situationen Schutzmassnahmen zu intensivieren (... und nicht zu lockern) - handle es sich um Expositionsverringerung wie bei Asbest, Luftreinhaltemassnahmen, UV-Prophylaxe oder Lärmschutzwände. Die geplante Revision der Verordnungen im Kernenergiebereich sieht dagegen sinnwidrig eine massive Erhöhung der Strahlengrenzwerte in einer Phase zunehmender Risiken durch Alterung der Kernkraftwerke vor. Zudem ist die Einwohnerzahl um die KKW seit der Bewilligung und Inbetriebnahme der Anlagen stark angestiegen. Dieses Vorgehen widerspricht nicht nur verantwortungsbewusstem ärztlichem Denken, sondern jegli- cher Vernunft. Wenn schon an eine Revision gedacht wird, wäre sie unter Berücksichtigung neuester wissenschaftlicher Erkenntnisse viel eher im Sinne einer Verschärfung der Strahlenschutzmassnahmen zu gestalten. Denn die aktuell geltenden Grenzwerte tragen der besonders ausgeprägten Strahlenempfindlichkeit von Kindern nicht genügend Rechnung.

\section{Résumé}

Die Revision der Verordnungen im Kernenergiebereich, die ab 1.1.2019 in Kraft treten soll, ist aus ärztlicher Sicht klar abzulehnen, da sie den Strahlenschutz der Bevölkerung im Vergleich zur aktuell geltenden gesetzlichen Regelung bei einem nicht einmal sehr seltenen Störfall in einem Schweizer Kernkraftwerk massiv schwächt. Nicht nur wissenschaftlich, sondern auch ethisch-moralisch begründete Argumente mit Blick auf die jetzt lebenden und die kommenden Generationen verpflichten uns zu dieser Stellungnahme. Wir rufen die Eidgenössische Strahlenschutzbehörde ENSI und den Bundesrat deshalb dringend zu verantwortungsvollem Handeln zugunsten der Sicherheit der unter ihren Schutz gestellten Bevölkerung auf.

Disclosure statement

Alle Autoren geben bekannt, dass keine Interessenkonflikte bestehen.

\section{Literatur}

Die vollständige Literaturliste finden Sie in der Online-Version des Artikels unter www.saez.ch 


\section{Literatur}

1 Revisionsvorlage (Teilrevision der Kernenergieverordnung, der Kernenergiehaftpflichtverordnung, der Ausserbetriebnahmever ordnung sowie der Gefährdungsannahmenverordnung) https:// www.admin.ch/ch/d/gg/pc/ind2018.html\#UVEK

2 Ozasa K, Shimizu Y, Suyama A, et al. Studies of the Mortality of Atomic Bomb Survivors, Report 14, 1950-2003: An Overview of Cancer and Noncancer Diseases. Radiat Res. 2012;177:229-243 http:// www.rerf.jp/library/rr_e/rr1104.pdf

3 National Research Council. Health Risks from Exposure to Low Levels of Ionizing Radiation, BEIR VII Phase 2 (2006): http://dels. nas.edu/resources/static-assets/materials-based-on-reports/reports-in-brief/beir vii final.pdf

4a Wirksamer Schutz gegen die Risiken ionisierender Niedrigstrahlung: Kenntnisstand betreffend Risiken ionisierender Strahlung im Niedrigdosisbereich; Medienmitteilung des Bundesrates vom 2. März 2018. https://www.bag.admin.ch/bag/de/home/themen/ mensch-gesundheit/strahlung-radioaktivitaet-schall/strahlung gesundheit/risiken-ionisierender-niedrigstrahlung.html

$4 \mathrm{~b}$ Protection efficace contre les risques des radiations ionisantes à faibles doses: L'état des connaissances sur les risques des radiations ionisantes à faibles doses; Communiqué du Conseil fédéral du 2 mars 2018. https://www.bag.admin.ch/bag/fr/home/themen/ mensch-gesundheit/strahlung-radioaktivitaet-schall/strahlunggesundheit/risiken-ionisierender-niedrigstrahlung.html

5 Richardson B, Cardis E, Daniels RD, et al. Risk of cancer from occupational exposure to ionising radiation: retrospective cohort study of workers in France, the United Kingdom, and the United States (INWORKS). BMJ 2015;351:h5359. http://www.bmj.com/content/351/bmj.h5359

6 Leuraud K, Richardson DB, Cardis E, et al. Ionising radiation and risk of death from leukaemia and lymphoma in radiation-monitored workers (INWORKS): an international cohort study. Lancet Haematol. 2015;2(7):e276-e281. http://www.thelancet.com/pdfs/ journals/lanhae/PIIS2352-3026(15)00094-0.pdf

7 Gillies M, Richardson B, Cardis E, et al. Mortality from Circulatory Diseases and other Non-Cancer Outcomes among Nuclear Workers in France, the United Kingdom and the United States (INWORKS). Radiat Res. 2017;188(3):276-90. https://www.ncbi.nlm.nih.gov/ pubmed/28692406

8 Darby S, Hill D, Auvinen A, et al. Radon in homes and risk of lung cancer: collaborative analysis of individual data from 13 European case-control studies. Brit Med J. 2005;330:223. http://www.bmj. com/content/330/7485/223

9 Pearce MS, Salotti JA, Little MP, et al. Radiation exposure from CT scans in childhood and subsequent risk of leukaemia and brain tumours: a retrospective cohort study. Lancet 2012;380:499-505. http://www.thelancet.com/pdfs/journals/lancet/PIISO1406736(12)60815-0.pdf

10 Mathews JD, Forsythe AV, Brady Z, et al. Cancer risk in 680000 people exposed to computed tomography scans in childhood or adolescence: data linkage study of 11 million Australians. Brit Med J. 2013;346:f2360. http://www.bmj.com/content/346/bmj.f2360

11 Kendall GM, Little MP, Wakeford R, et al. A record-based case-control study of natural background radiation and the incidence of childhood leukaemia and other cancers in Great Britain during 1980-2006. Leukemia. 2013;27:3-9. https://www.ncbi.nlm.nih.gov/ pmc/articles/PMC3998763/

12 Spycher BD, Lupatsch JE, Zwahlen M, et al. for the Swiss Pediatric Oncology Group and the Swiss National Cohort Study Group. Background Ionizing Radiation and the Risk of Childhood Cancer: A Census-Based Nationwide Cohort Study. Environ Health Perspect. 2015;123:622-8. https://ehp.niehs.nih.gov/1408548/
13 Executive Order 13777, «Enforcing the Regulatory Reform Agenda», by President Donald Trump, 24th February 2017. https://www.gpo. gov/fdsys/pkg/DCPD-201700139/pdf/DCPD-201700139.pdf

14 Cuttler JM, Hannum WH. Current radiation protection limits: An urgent need for change. Nuclear News. 2017;60(10):34-8. http:// radiationeffects.org/wp-content/uploads/2017/09/CuttlerHannum-2017Sep_NN_Current-rad-protect-limits-C.pdf

15 Siegel JA, Pennington CW, Sacks B, et al. The Birth of the Illegitimate Linear No-Threshold Model: An Invalid Paradigm for Estimating Risk Following Low-dose Radiation Exposure. Am J Clin Oncol. 2018;41(2):173-7. https://www.ncbi.nlm.nih.gov/pubmed/26535990

16 Siegel JA, Pennington CW, Sacks B. Subjecting Radiologic Imaging to the Linear No-Threshold Hypothesis: A Non Sequitur of Non-Trivial Proportion. J Nucl Med. 2017;58(1):1-6. https://www.ncbi.nlm. nih.gov/pubmed/27493264

17 Cuttler JM, Feinendegen LE, Socol Y. Evidence that Lifelong Low Dose Rates of Ionizing Radiation Increase Lifespan in Long- and Short-Lived Dogs. Dose-Response. 2017;15(1):1-6. www.ncbi.nlm. nih.gov/pmc/articles/PMC5347275/

18 Calabrese EJ. LNTgate: The Ideological History of Cancer Risk Assessment. Toxicol Res Applic. 2017;1-3. http://journals.sagepub. com/doi/10.1177/2397847317694998

19 Reichmuth A. Länger leben dank Radioaktivität. Basler Zeitung vom 11.1.2018. https://bazonline.ch/wissen/natur/laenger-lebendank-radioaktivitaet/story/22689973

20a Bei einem 10000-jährlichen Naturereignis müssen die KKW die 100-mSv-Grenze einhalten (ENSI-Homepage, News, 1. Feb. 2018). https://www.ensi.ch/de/2018/02/01/bei-einem-10000-jaehrlichennaturereignis-muessen-die-kkw-die-100-msv-grenze-einhalten/

20b Les centrales nucléaires doivent tenir la valeur limite de $100 \mathrm{mSv}$ pour un événement naturel se passant tous les 10000 ans (ENSIHomepage, News, 5. Feb. 2018). https://www.ensi.ch/fr/2018/02/05/ centrales-nucleaires-doivent-tenir-valeur-limite-de-100-msvevenement-naturel-se-passant-10-000-ans/?noredirect=fr_FR

21 Empfehlungen der International Commission of Radiological (ICRP), Publication 103, 2007. http://doris.bfs.de/jspui/bitstream/ urn:nbn:de:0221-2009082154/1/BfS_2009_BfS-SCHR-47-09.pdf

22 "smarter medicine»: die "Top-5-Liste» der Schweizerischen Gesellschaft für Chirurgie (SGC). Schweiz Ärztezeitung. 2018;99(1-2):12-3. https://saez.ch/de/article/doi/saez.2018.06342/

23 Little MP, Azizova TV, Bazyka D, et al. Systematic Review and Metaanalysis of Circulatory Disease from Exposure to Low-Level Ioni zing Radiation and Estimates of Potential Population Mortality Risks. Environ Health Perspect. 2012;120:1503-11. https://ehp.niehs. nih.gov/1204982/

24 Information 21.3.2018; Kanton Aargau, Departement Gesundheit und Soziales. www.ag.ch/dgs

25 Stellungnahme des ENSI zum deterministischen Nachweis des KKB zur Beherrschung des 10000 -jährlichen Erdbebens; 7.7.2012. http://static.ensi.ch/1341818977/kkb_sn-erdbebennachweis_final_ geschwaerzt.pdf

26 Antwort zu Frage 18.5204 Munz (Fragestunde Nationalrat vom 12.3.2018). https://www.parlament.ch/centers/documents/de/ fragestunde-2018-03-12-antworten.pdf

27 ENSI. Anforderungen an die deterministische Störfallanalyse für Kernanlagen: Umfang, Methodik und Randbedingungen der technischen Störfallanalyse, Ausgabe Juli 2009, Erläuterungsberich zur Richtlinie ENSI-AO1/d, S.5, Abschnitt 2.4.1. http://static.ensi. ch/1313045150/a-001_d_erlaueterungsbericht.pdf 